\section{CDC Recommendation Clarified}

\section{To The Editor:}

In the article, "Multidose medication vial sterility: An in-use study and a review of the literature," ${ }^{1}$ Longfield et al state: "Although no formal guidelines have been promulgated, the Centers for Disease Control (CDC) had suggested to some pharmacies that MDV be discarded within 24 hours of opening." There is a need to clarify current CDC recommendations in this area.

It is true that CDC had made this suggestion in the past. However, in October 1981 CDC published the Guideline for Hospital Environmental Control ${ }^{2}$ which contained a section on the pharmacy. Recommendation 6 in this section states:

Single-use (single-dose) containers (vials) should be used for admixture whenever possible. When multipleuse containers intended for intravenous or intramuscular use are opened, they should be marked with the date and time that the container is entered. The product label or package insert should be consulted to determine if refrigeration of the container is necessary. (The proper storage temperature is product-specific and is determined by many factors such as stability of ingredients and optimal activity of antibacterial preservatives; bacteria survival in some containers may be enhanced by refrigeration. Unless an expiration date is stated on the product label or package insert, it is not known if multiple-use containers, once entered, should be discarded after a specific or arbitrary length of time.)

Longfield et al recommend that multiple-dose medication vials be dated when opened and, unless contamination is apparent or suspected, that the vials be discarded either when empty or on the expiration date set by the manufacturer. These recommendations are consistent with current CDC recommendations.

\section{REFERENCES}

1. Longfield R, Longfield J, Smith LP, et al: Multidose medication vial sterility: An in-use study and a review of the literature. Infect Control 1984; 5(4):165-169.

2. Simmons BP, Hooton TM, Mallison GF: Guideline for hospital environmental control. Infect Control 1982; 3(1):59-60.

Julia S. Garner, RN, MN

Walter W. Williams, MD, MPH

James M. Hughes, MD

Hospital Infections Program Center for Infectious Diseases

Centers for Disease Control Atlanta, Georgia

\section{Comments on} "Nosocomial Sexually
Transmitted Diseases"

\section{To the Editor:}

Dr. Rein's Editorial regarding Nosocomial Sexually Transmitted Diseases $^{1}$ presents a considerable amount of important information and a frequent recommendation to apply body discharge precautions. However, by recommending these precautions on a disease-specific basis, we may be encouraging a less than desirable standard of general practice.

Frankly, when would "drainage," "secretion," "discharge" or "blood/ body fluid" precautions not be prudent? We know that a wide variety of infections can be transmitted in the absence of visible mucosal lesions, that herpetic whitlow is an occupational hazard in nursing, that most hepatitis carriers will not be recognized at the time of exposure. Why would anyone fail to wear gloves for direct contact with genitals, mucous membranes, lesions or discharges of any patient?

We should reserve additional precaution or "isolation" regimes for measures over and above normal hygiene. We should not encourage staff to "isolate" patients when simple hygiene would suffice, in part because this may tend to give a false sense of security when failing to observe those hygienic measures with unlabelled patients. Gloves should be worn for all suctioning, mouth care, catheter insertion, perineal care, etc., regardless of diagnosis. This would not only provide better protection for staff members, but also improve their understanding of infectious disease epidemiology. That, in turn, can help them to counsel and reassure their patients. A patient with genital herpes may find it more reassuring to learn that certain precautions are taken for genital contact with every patient as opposed to being singled out for "isolation;" other patients on the same ward may find it more reassuring to know that precautions are taken reliably rather than reactively.

The message to laboratory workers that all biological materials should be treated with caution is well-established. The concept that all surgical cases should be treated in one manner, and not arbitrarily divided into "clean" and "dirty" cases, is also wellestablished. It is now time to bring a similar message to ward staffs!

We can avoid the cost and negative connotations of "isolation" for many patients, reduce the number of different protocols staff are expected to remember, and improve the level of safety for both staff and patients. We could avoid admitting "It would seem that our profession still tends to avoid 